\title{
Avaliação epidemiológica das regiões do Brasil na pandemia de COVID-19
}

\author{
Epidemiological evaluation of the regions of Brazil in the COVID-19 pandemic
}

Evaluación epidemiológica de las regiones de Brasil en la pandemia COVID-19

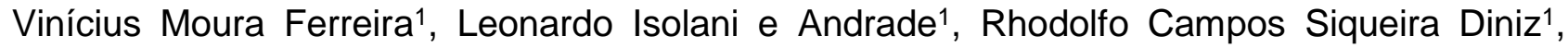
Eduarda de Oliveira Karklins², Letícia Maria Pereira de Miranda1, Lucas Henrique Pereira Ramos $^{1}$, Paulo José Soares André Oliveira ${ }^{1}$, Tamara Rodrigues Fonseca Souza ${ }^{1}$, Vinícius Silva Belo ${ }^{1}$, Saulo Nascimento de Melo ${ }^{1 *}$.

\section{RESUMO}

Objetivo: Analisar os dados da COVID-19 entre as regiões do Brasil, comparando as realidades distintas, levando em consideração as taxas de mortalidade, letalidade, incidência e isolamento social. Métodos: Trata-se de um estudo observacional analítico. Foram analisadas a partir da densidade demográfica, as taxas de incidência, mortalidade, letalidade e isolamento social. Ademais, foram levantados dados referentes ao número de leitos público e privados disponíveis para COVID-19 em cada estado do país. Para análise das associações foi utilizado a correlação de Spearman entre as variáveis densidade populacional, número de casos, número de leitos e quantidade de óbitos nos estados brasileiros. Resultados: O estudo avaliou 3.817.904 casos confirmados e 120.530 óbitos em todo o Brasil, sendo que a letalidade geral foi de 3,15. A Região Norte apresentou as maiores taxas de incidência e a região Sul apresentou as menores taxas de incidência. Com esses dados, foi possível verificar a existência de uma relação positiva entre as taxas de incidência e mortalidade. Conclusão: $O$ estudo demonstrou uma enorme discrepância epidêmica e socioeconômica entre as regiões brasileiras, implicando diretamente na incidência e mortalidade por COVID-19.

Palavras-chave: Covid-19, Pandemia, Epidemiologia, Sars-Cov-2, Brasil.

\begin{abstract}
Objective: Analyze the data from COVID-19 between the regions of Brazil, comparing the different realities, taking into account the mortality, lethality, incidence and social isolation rates. Methods: This is an observational analytical study. From the demographic density, the rates of incidence, mortality, lethality and social isolation were analyzed. In addition, data were collected regarding the number of public and private beds available for COVID-19 in each state of the country. Spearman's correlation between the variables population density, number of cases, number of beds and number of deaths in the Brazilian states was used to analyze the associations. Results: The study evaluated 3,817,904 confirmed cases and 120,530 deaths across Brazil, with a general lethality of 3.15. The North Region had the highest incidence rates and the South Region had the lowest incidence rates. With these data, it was possible to verify the existence of a positive relationship between incidence and mortality rates. Conclusion: The study demonstrated a huge epidemic and socioeconomic discrepancy between Brazilian regions, directly impacting the incidence and mortality from COVID-19.
\end{abstract}

Keywords: Covid-19, Pandemic, Epidemiology, Sars-Cov-2, Brazil.

1 Universidade Federal de São João del-Rei (UFSJ), Divinópolis - MG. *E-mail: saulomelobio@hotmail.com

2 Pontifícia Universidade Católica de Minas Gerais (PUC Minas), Betim - MG.

SUBMETIDO EM: 3/2021

ACEITO EM: 4/2021

PUBLICADO EM: 4/2021 


\section{RESUMEN}

Objetivo: Analizar los datos de COVID-19 entre las regiones de Brasil, comparando las diferentes realidades, tomando en cuenta las tasas de mortalidad, letalidad, incidencia y aislamiento social. Métodos: Este es un estudio analítico observacional. A partir de la densidad demográfica se analizaron las tasas de incidencia, mortalidad, letalidad y aislamiento social. Además, se recopilaron datos sobre la cantidad de camas públicas y privadas disponibles para COVID-19 en cada estado del país. Para analizar las asociaciones se utilizó la correlación de Spearman entre las variables densidad de población, número de casos, número de camas y número de defunciones en los estados brasileños. Resultados: El estudio evaluó 3.817.904 casos confirmados y 120.530 muertes en todo Brasil, con una letalidad general de 3,15. La Región Norte tuvo las tasas de incidencia más altas y la Región Sur tuvo las tasas de incidencia más bajas. Con estos datos se pudo comprobar la existencia de una relación positiva entre las tasas de incidencia y mortalidad. Conclusión: El estudio demostró una enorme discrepancia epidémica y socioeconómica entre las regiones brasileñas, lo que repercutió directamente en la incidencia y la mortalidad por COVID-19.

Palabras clave: Covid-19, Pandemia, Epidemiología, Sars-Cov-2, Brasil.

\section{INTRODUÇÃO}

Os coronavírus são uma família de vírus que causam infecções respiratórias. A COVID-19, causada por um vírus dessa família, foi descoberta em 31 de dezembro de 2019 em Wuhan, China (HONGZHOU L, et al., 2020). Uma semana depois, as autoridades chinesas identificaram um novo coronavírus, que foi nomeado SARS-CoV-2 (HUI DS, et al., 2020). Três meses após a descoberta da doença, a Organização Mundial da Saúde (OMS) declarou que os surtos de coronavírus constituem uma emergência de saúde pública de importância internacional e listou o COVID-19 como uma pandemia (OPAS/OMS, 2020).

O SARS-CoV-2 tem alta transmissibilidade e provoca uma síndrome respiratória aguda que varia de casos leves, responsáveis por cerca de $80 \%$, a casos severos com insuficiência respiratória, representando $5 \%$ a $10 \%$ dos casos. O método de transmissão ocorre através do contágio direto, como um aperto de mão, gotículas de saliva, espirro, tosse ou fômites (KUCHARSKI AJ, et al., 2020). Nos casos leves, deve-se manter o paciente em isolamento domiciliar, com monitoramento do estado de saúde e, nos casos mais graves, a conduta é a hospitalização do paciente, com suporte ventilatório nas unidades de internação ou de terapia intensiva, a julgar pela gravidade do caso (BRASIL, 2021).

O primeiro caso de Covid-19 no Brasil foi confirmado em fevereiro de 2020, quase dois meses após o diagnóstico do primeiro caso na China. A transmissão comunitária da Covid-19 foi reconhecida em todo o território nacional em 20 de março de 2020 (OLIVEIRA WK, et al., 2020). No país, foi sancionada a Lei 13.979, que dispõe sobre as medidas para enfrentamento da epidemia da COVID-19 e elenca as intervenções não farmacológicas comunitárias (INF's) que podem ser adotadas. As Unidades da Federação (UF's) passaram a adotar tais medidas a partir da segunda semana de março de 2020 e, posteriormente, aprovaram novos decretos estabelecendo medidas mais rigorosas (GARCIA LP e DUARTE E, 2020).

A ausência de imunidade prévia de toda a humanidade, somada à elevada infectividade do SARS-CoV2, bem como a inexistência de vacina contra este vírus, faz com que o crescimento do número de casos seja exponencial. Nesse caso, foram apontadas intervenções não farmacológicas (INF's), destinadas a suprimir a transmissão de pessoa para pessoa, com objetivo de retardar a disseminação da doença e, assim, reduzir ou atrasar o pico de incidência da curva epidêmica (LANA CNA, et al., 2020).

As INF's são medidas de saúde pública com alcance individual, ambiental e comunitário. Medidas individuais incluem lavar as mãos, usar máscaras e manter o distanciamento social. As medidas ambientais referem-se à aeração e exposição ao sol do ambiente e limpeza de rotina do ambiente e das superfícies, processos que ajudam a eliminar o vírus. As medidas comunitárias são ações tomadas por gestores, empregadores e/ou líderes comunitários para proteger a população, no qual incluem a restrição ao 
funcionamento de escolas, universidades, locais de convívio comunitário, transporte público, além de outros locais onde haja aglomeração de pessoas, como serviços não essenciais (GARCIA LP e DUARTE E, 2020; JÚNIOR CAM, 2020).

O Brasil possui cinco regiões geográficas: Norte, Nordeste, Centro-Oeste, Sudeste e Sul. Essas possuem inúmeras diferenças geográficas e econômicas. A região Norte, a maior em extensão geográfica, conta com sete estados e possui o menor PIB (Produto Interno Bruto) e o segundo menor PIB per capita entre as regiões do país (BRASIL, 2010).

A região Nordeste é a segunda região mais populosa do país, mas é a terceira mais povoada. Essa região possui a terceira maior extensão territorial e o terceiro maior PIB, apesar de ser a região com o menor PIB per capita do país. A região Centro-Oeste é a segunda maior do país em extensão territorial, sendo também a região menos populosa e a segunda menos povoada. Apresenta o maior PIB per capita, apesar de apresentar o segundo menor PIB entre as demais regiões do país (BRASIL, 2010).

A região Sudeste é a mais populosa e povoada do país, apresentando também o maior PIB e segundo maior PIB per capita. A região Sul possui a menor extensão territorial entre as regiões do país. Além disso, essa região apresenta a segunda maior densidade demográfica e o segundo maior PIB do país (BRASIL, 2010).

Nesse contexto, é fundamental a atuação do Sistema Único de Saúde (SUS) e das demais áreas do sistema de proteção social de forma articulada, de modo a se favorecer a adesão das pessoas às intervenções não farmacológicas e minimizar os impactos deletérios das medidas comunitárias. A proteção da saúde pública deverá ser norteadora pelas decisões a serem tomadas pelos gestores. Essas devem ser baseadas nas melhores evidências disponíveis e comunicadas de forma transparente, para promover a confiança da população (GARCIA LP e DUARTE E, 2020).

Diante disso, o objetivo do presente estudo é apresentar os dados a respeito da COVID-19 entre as regiões do Brasil, além de comparar as estratégias de controle adotadas. Foram avaliadas as taxas de mortalidade, letalidade, incidência e isolamento social em cada região.

\section{MÉTODOS}

Estudo observacional analítico, conduzido com base em dados secundários das semanas epidemiológicas (SE) 9 a 35, que corresponde ao período de estudo do dia 23/02/2020 até o dia 29/08/2020, extraídos dos boletins epidemiológicos sobre a COVID-19 das Secretarias Estaduais de Saúde (SES) de todas as UF do Brasil. Esses boletins são fonte de dados oficiais do país e apresentam informações completas das variáveis analisadas.

A análise da situação epidemiológica das regiões baseou-se em dados do número de casos confirmados e do número de óbitos, coletados por meio dos sites das Secretarias Estaduais de Saúde das Unidades Federativas do país. Além disso, foram coletados dados referentes ao número de leitos públicos e privados, distribuídos por regiões, obtidos através do Cadastro Nacional de Estabelecimentos de Saúde (CNES) de junho de 2020. As informações relativas ao distanciamento social foram coletadas por meio de dados de operadoras telefônicas nacionais, disponibilizados no site "Mapa Brasileiro da COVID in Loco" (INLOCO, 2020).

Para o cálculo da taxa de incidência da COVID-19 por regiões do Brasil, foi utilizado no numerador o número de casos de COVID-19 detectado em cada SE do estudo e no denominador, o número total da população, multiplicada por cem mil habitantes, considerando a projeção do Instituto Brasileiro de Geografia e Estatística (IBGE) para 2020.

No cálculo da taxa de mortalidade da COVID-19, foi utilizado como numerador, o número de óbitos de COVID-19 detectados em pessoas das regiões, em cada SE do estudo. No denominador, foi utilizado o número total da população em cada região, multiplicada por cem mil.

No cálculo da taxa de letalidade da COVID-19, foi utilizado como numerador, o número de óbitos de COVID-19 detectados em pessoas das regiões, em cada SE do estudo. No denominador, foi utilizado o número total da população infectado, multiplicada por cem. 
Para o cálculo da densidade populacional utilizou-se o número de habitantes por região dividido pela área total de cada região. Para analisar as associações, foi utilizado a Correlação de Spearman entre as variáveis densidade populacional, número de casos, número de leitos e a quantidade de óbitos nos estados do Brasil. Todas as análises estatísticas foram realizadas no software SPSS (IBM, 2017).

Este estudo foi realizado a partir de um banco de dados secundários. Nesse contexto, não foi necessária a submissão ao Comitê de Ética em Pesquisa (CEP) por seguimento das normas éticas do País, de acordo com a Resolução do Conselho Nacional de Saúde oㅜ 510, de 7 de abril de 2016.

\section{RESULTADOS}

Durante o período estudado, foram avaliados 3.817 .904 casos confirmados e 120.530 óbitos em todo o território nacional, sendo que a letalidade geral foi de 3,15. A Região Norte apresentou as maiores taxas de incidência, com pico na semana 26. As regiões Sudeste e Nordeste apresentaram curvas semelhantes e a região Sul teve as menores taxas de incidência. A região Centro-Oeste apresentou taxas semelhantes às da região Sul. Porém, a partir da semana 21, houve aumentos expressivos nas taxas de incidência dessa região (Gráfico 1).

No Gráfico 2 é possível notar a taxa de mortalidade por regiões do Brasil. A partir da $13^{a}$ semana, houve um aumento dessa taxa na região Sudeste. A região Norte apresentou uma curva ascendente, com início na semana 14 , com o pico na $19^{\text {a }}$ semana. A inclinação descendente nessa região ocorreu após a $20^{a}$ semana. Além disso, essa região apresentou a maior taxa de mortalidade no território nacional. A região Sudeste aparece em seguida, com uma inclinação ascendente mais branda, sendo que o pico ocorreu na $25^{a}$ semana, quando registrou números maiores que as demais regiões. A região Centro-Oeste apresentou a segunda menor inclinação da curva e, até a semana 24, apresentava o menor número de óbitos (835).

A partir da semana 19 houve um aumento na taxa de mortalidade nessa região, sendo que na $25^{a}$ semana o Centro-Oeste ultrapassou a Região Nordeste no número de óbitos por habitantes. A Região Nordeste iniciou uma constante a partir da $14^{\mathrm{a}}$ semana e se manteve praticamente constante a partir da $19^{\mathrm{a}}$ semana. A região Sul é a que apresentou a curva menos inclinada e com o menor número absoluto de óbitos durante todo o período analisado, entretanto, na segunda metade do período analisado (SE 22 à SE 35) houve um aumento da taxa de mortalidade dessa região (Gráfico 2).

Gráfico 1 - Taxa de Incidência por Regiões do Brasil.

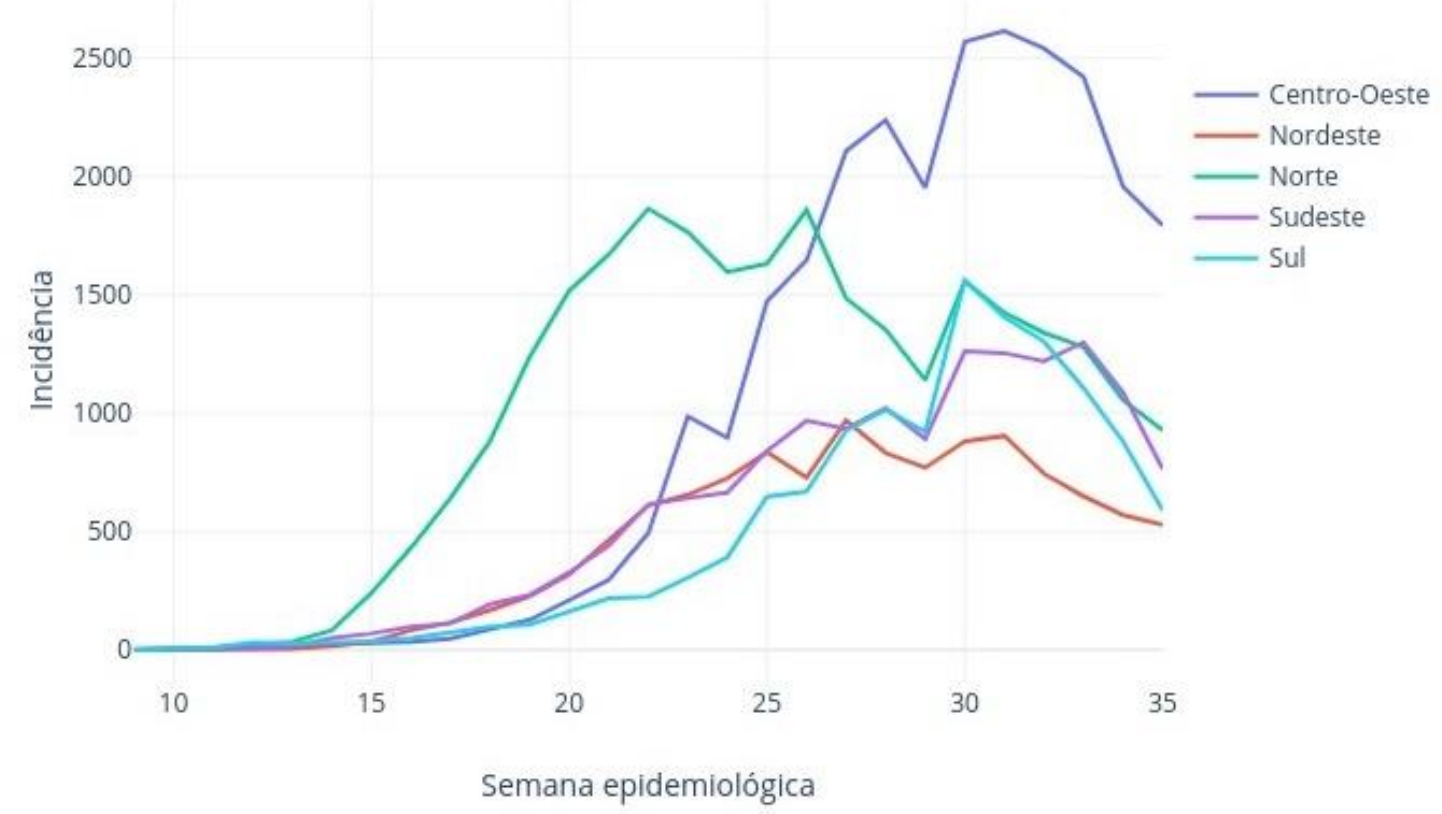

Fonte: Ferreira VM, et al., 2021; dados extraídos das Secretarias Estaduais de Saúde (SES). 
Gráfico 2 - Taxa de Mortalidade por Regiões do Brasil (por 100.000 habitantes).

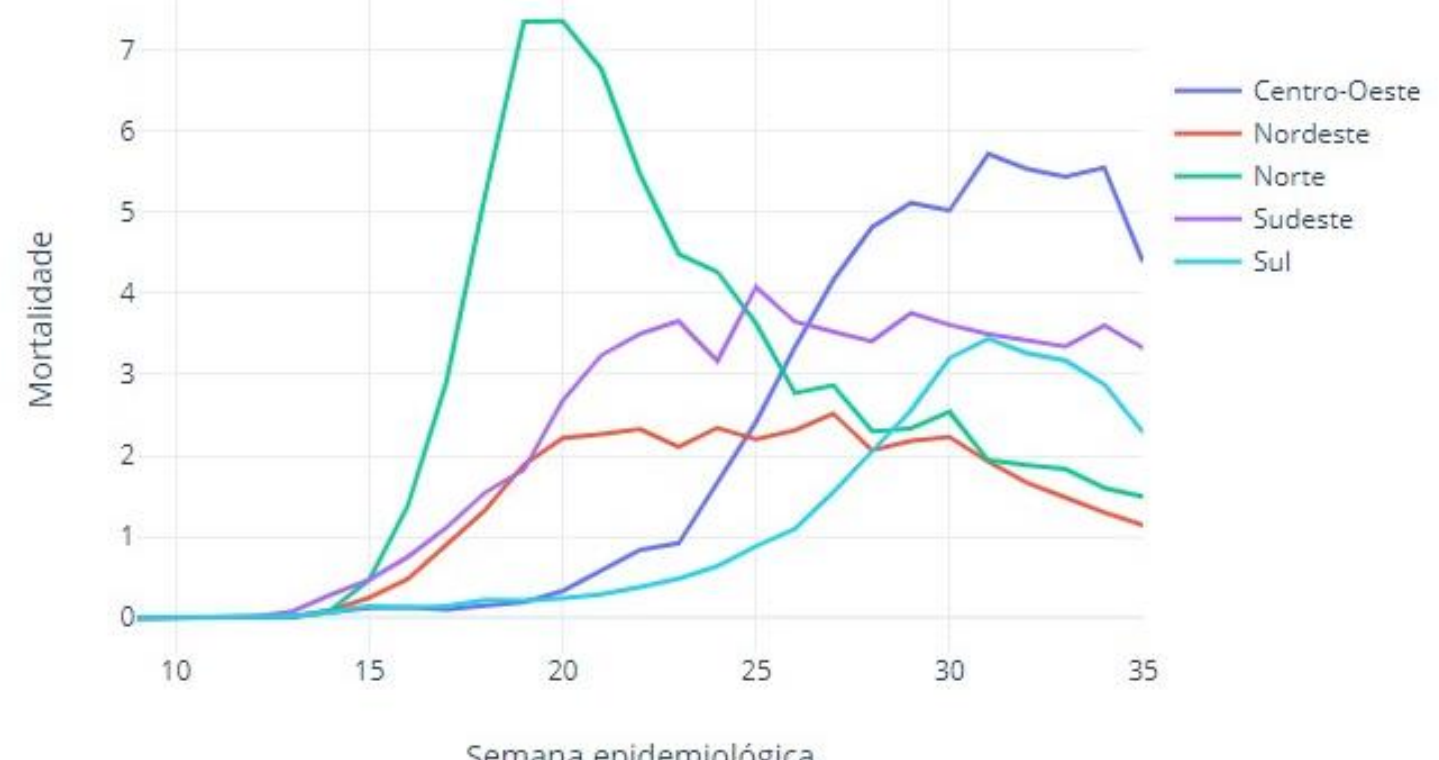

Semana epidemiológica

Fonte: Ferreira VM, et al., 2021; dados extraídos das Secretarias Estaduais de Saúde (SES).

De modo geral, houve maiores taxa de letalidade a partir da SE 12. Considerando todo o período estudado, as regiões Sudeste e Nordeste apresentaram as maiores taxas. As regiões Sul e Centro-Oeste apresentaram os menores índices entre a semana 16 e a semana 25, sendo que essas duas regiões se alternaram nas menores taxas. A Região Norte teve uma curva ascendente a partir da semana 13 e atingiu seu pico na semana 18, após isso houve quedas nas taxas, assim como as demais regiões. Entretanto, as regiões Norte e Nordeste mantiveram um pico em forma de platô, que perdurou por cerca de duas semanas, enquanto as demais regiões apresentaram picos mais agudos e com menor duração (Gráfico 3).

Gráfico 3 - Taxa de Letalidade por Regiões do Brasil.

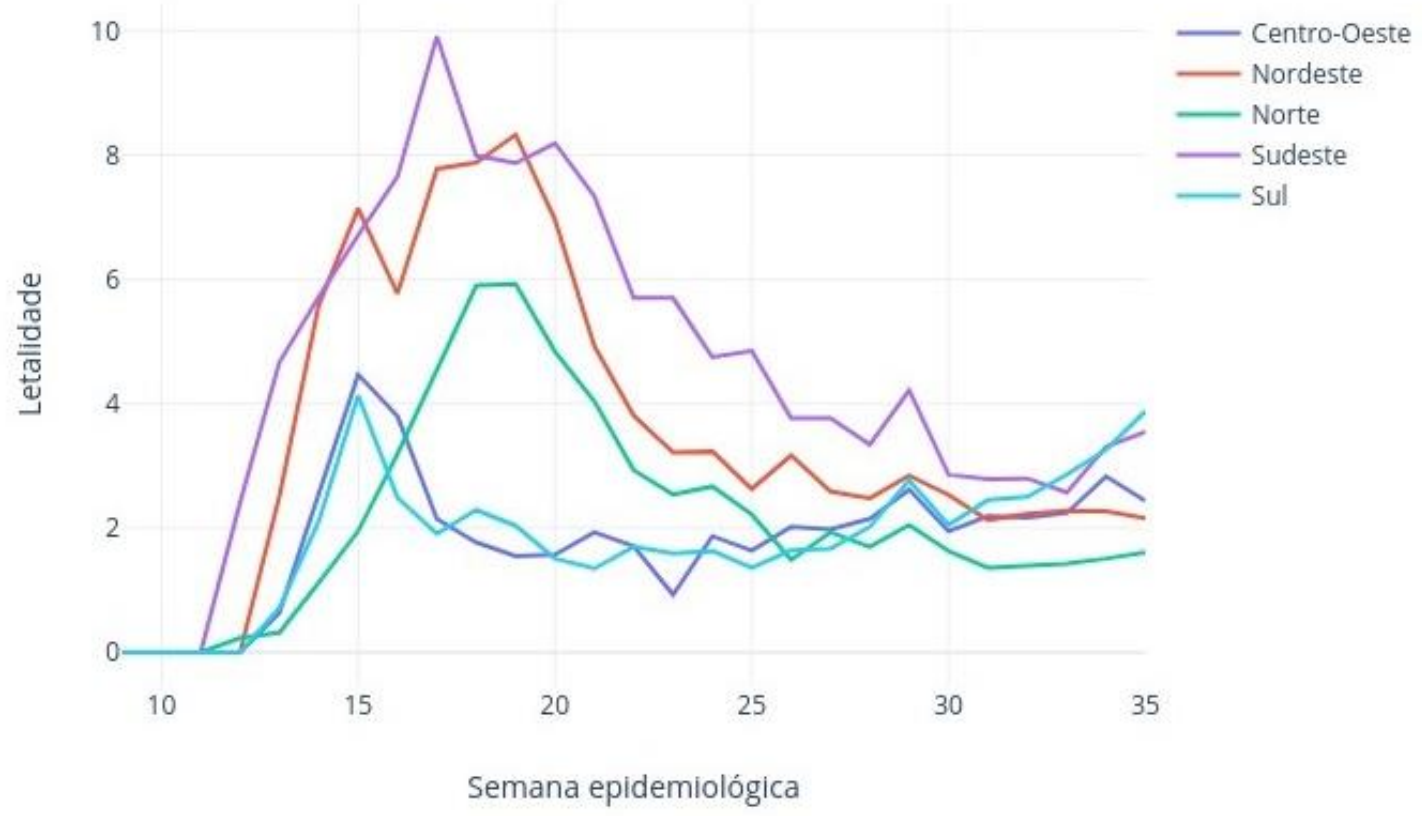

Fonte: Ferreira VM, et al., 2021; dados extraídos das Secretarias Estaduais de Saúde (SES)

Os dados obtidos através do controle das operadoras telefônicas mostram que, entre as semanas 11 e 13 , houve um considerável aumento da adesão ao isolamento social em todas as regiões. O pico de 
isolamento social ao longo do período de estudo foi evidenciado na semana 13. Da semana 13 até a semana 14 houve um decréscimo de cerca de $10 \%$, sendo que a região Sul manteve a maior porcentagem de isolamento das regiões e as regiões Norte e Centro-Oeste com as menores. A partir dessa semana até a semana 25 aconteceu um decréscimo mais lento de cerca de $8 \%$. A partir da semana 25 o percentual de isolamento social começou a oscilar entre 35 e 40\% (Gráfico 4).

Gráfico 4 - Taxa de Isolamento por Regiões do Brasil.

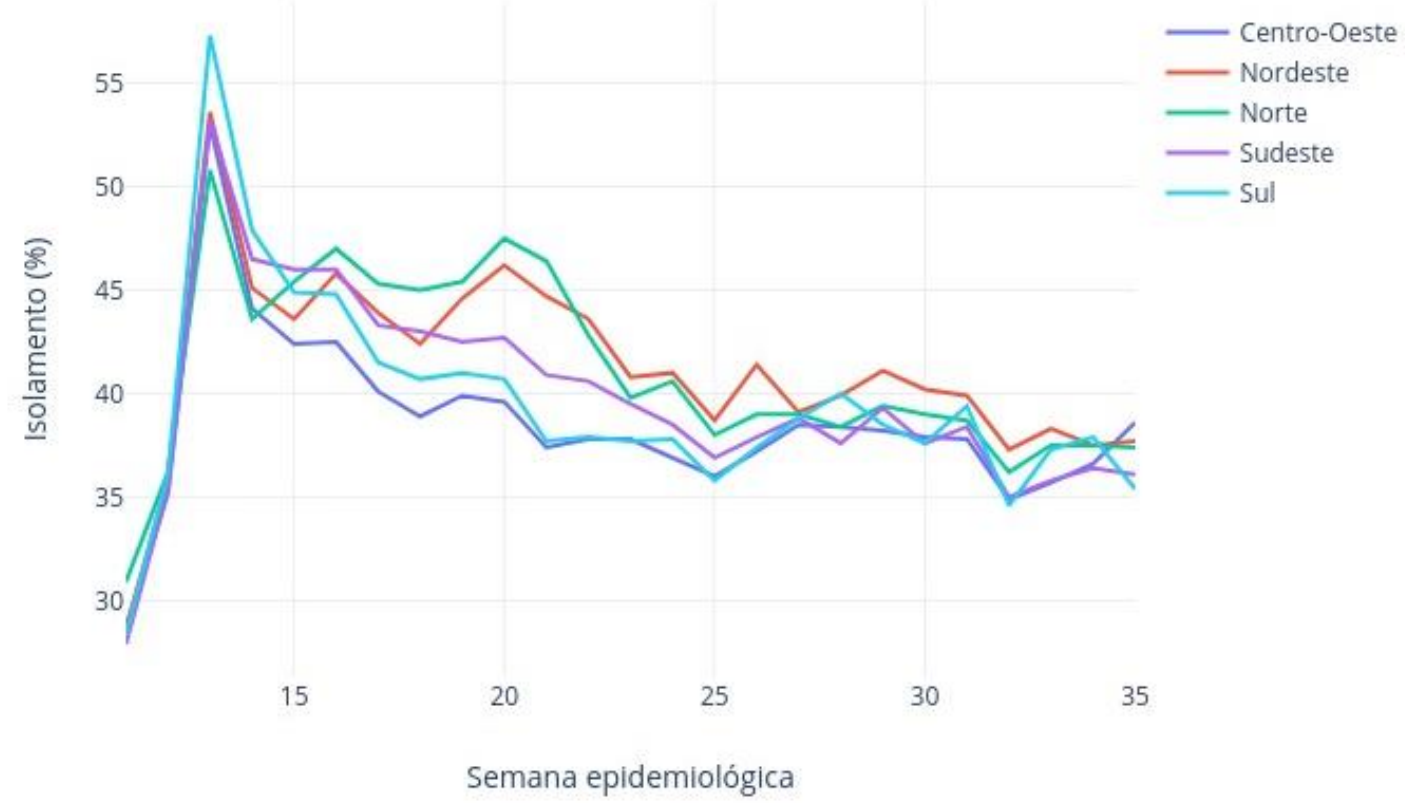

Fonte: Ferreira VM, et al., 2021; dados extraídos do site "Mapa Brasileiro da COVID in Loco" (INLOCO, 2020).

O Gráfico 5 mostra o total de leitos exclusivos para COVID por 100 mil habitantes, estratificado por leitos do SUS e privados. As regiões Sudeste e Centro-Oeste apresentavam a maior oferta de leitos, sendo 11,50 e 10,07 leitos para cada 100.000 habitantes, respectivamente. Já a região Norte apresentou a menor cobertura, com apenas 7,46 leitos para cada 100 mil habitantes. Já a região Sul apresentava a maior taxa de leitos disponíveis no SUS, sendo 5,18 leitos exclusivos para COVID.

Gráfico 5 - Total de Leitos Exclusivos para Covid-19 por Regiões Brasileiras (por 100.000 habitantes).

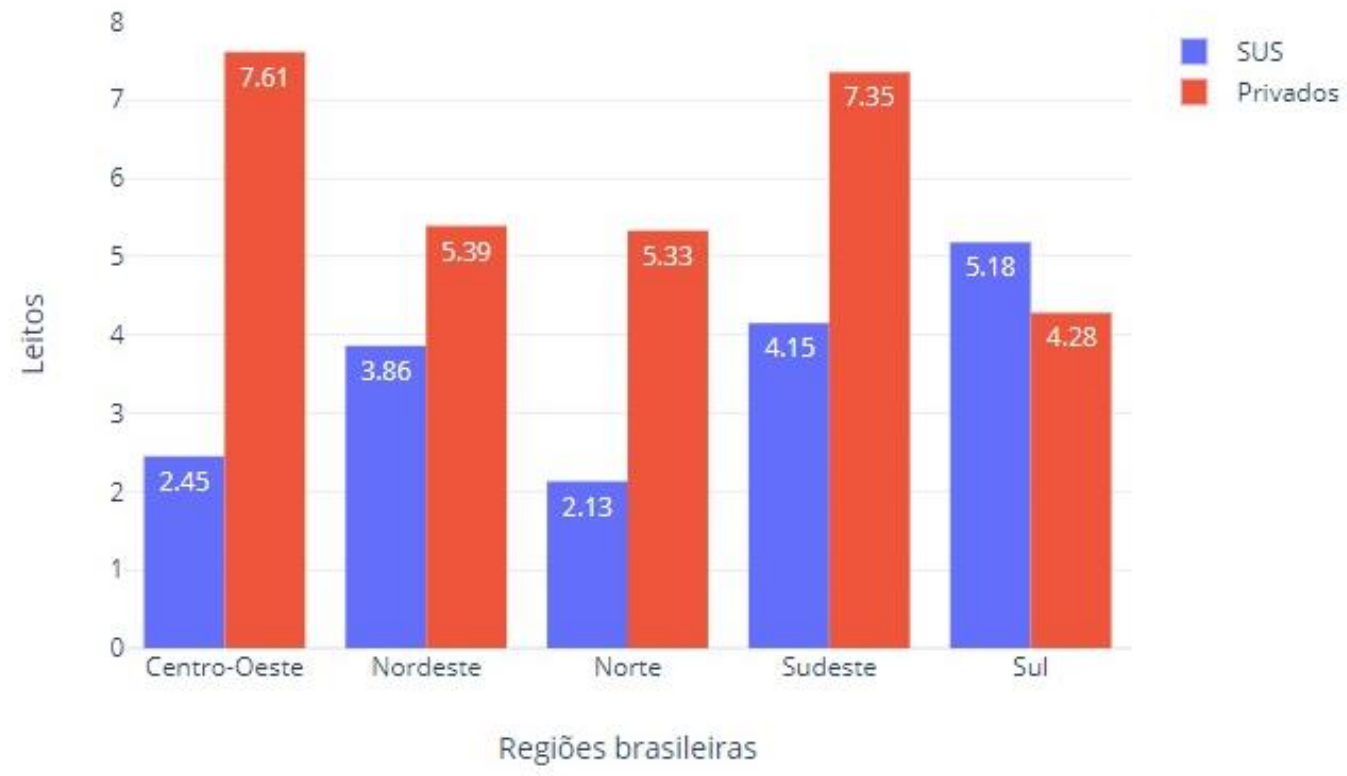

Fonte: Ferreira VM, et al., 2021; dados extraídos do DATASUS. 
De acordo com o teste de correlação de Spearman, os estados que possuíam mais leitos também apresentaram o maior número de óbitos $(r=0,8, p<0,05)$, além disso os estados com maiores taxas de densidade populacional, apresentaram mais número de casos $(r=0,5, p<0,05)$.

\section{DISCUSSÃO}

Durante o período do estudo, foi possível notar uma relação entre a incidência e a mortalidade, uma vez que essas taxas tiveram inclinação similar. A Região Norte apresentou os maiores números, com aumento da incidência e da mortalidade a partir da SE 15. Além disso, a Região Centro-Oeste apresentou um aumento da inclinação das curvas a partir da SE 23, se estendendo até a SE 29. As demais regiões também apresentaram uma relação positiva entre a incidência e a mortalidade durante o período de estudo, em um estudo realizado por Zhang CH e Schwartz GG (2020), também foram encontradas correlações positivas entre as taxas de incidência e mortalidade por COVID-19, nos Estados Unidos.

As Regiões Sudeste e Nordeste apresentaram curvas de incidência semelhantes até a SE 26, entretanto, a partir da SE 19 o Sudeste apresentou um aumento quando comparado com o Nordeste. Segundo o estudo de Noronha KVMS, et al. (2020), a distribuição de leitos públicos e privados nessas regiões, aliado ao fato de a Região Sudeste apresentar uma densidade demográfica consideravelmente maior que a Região Nordeste $(95,58$ para 36,77$)$, faz com que a demanda pela rotatividade de leitos seja maior.

A Região Sul apresentou as menores taxas de incidência e mortalidade até a SE 29, a partir desta semana a região passou a ocupar o terceiro lugar em ambas as taxas, e somente na SE 31, a partir da redução da curva de incidência, a região começou a reduzir também a inclinação da curva de mortalidade, mostrando existir uma relação positiva entre esses dois indicadores, conforme estudo feito por Zhang $\mathrm{CH}$ e Schwartz GG (2020).

Nesse estudo, o teste de Spearman demonstrou que o número de casos foi associado a densidade demográfica nas regiões do Brasil. De acordo com Leiva GC, et al. (2020), a propagação do vírus é mais rápida em cidades mais populosas, devido à dificuldade do isolamento e uso intensivo do sistema de transporte. Os trabalhadores mais carentes priorizam suas fontes de renda, principalmente quando os casos são mais leves, para sustentar sua família (LANA CNA, et al., 2020; FRANCÊS CRL, 2020).

Nesses casos, é comum que haja vários habitantes em uma residência, tornando o isolamento impossível de ser feito, o que causa uma ruptura nos protocolos que visam a quarentena (PEREIRA MD, 2020), como no caso das favelas, devido a desigualdade social predominante nesse ambiente, conforme descrito pelo Boletim Socioepidemiológico da COVID-19 nas Favelas (PERES AC, 2020).

O isolamento social é visto como a medida com maior eficácia, mesmo em cidades com propagação acelerada (PIRES LN, et al., 2020). Esse fator pode explicar o motivo da região Sul, mesmo com a $2^{2}$ maior densidade demográfica, ter um número de casos menor em comparação com as outras. No início da pandemia, a região apresentou a maior taxa de isolamento social do Brasil, o que ocasionou um menor do número de casos, aliado ao fato de que a região Sul possui o menor número de trabalhadores informais (GOUVEIA GPM, et al., 2020).

Desse modo, o isolamento na região Sul pôde apresentar um maior sucesso devido aos fatores socioeconômicos (FREITAS CM, et al., 2020). Esse fato pode ser evidenciado quando se observa que o número de casos era mais alto nas regiões que possuíam menor PIB per capita, como as regiões Norte e Nordeste, durante o início da pandemia. Após a liberação do Auxílio Emergencial, recurso disponibilizado pelo Governo Federal como forma de auxiliar o cidadão brasileiro, a população que, antes não teria condição financeira para manter o isolamento social, passou a ter a possibilidade de se isolar, conforme assinalado pelo estudo de Farias. Dessa forma, o número de casos nessas regiões começou a diminuir (FARIAS HS, 2020). 
As regiões com maior número de leitos públicos por habitante apresentaram uma taxa de mortalidade menor que as regiões com a menor proporção de leitos SUS. Como exemplo, pode-se citar as Regiões Norte e Nordeste, com os menores números de leitos públicos por habitantes e maiores taxas de mortalidade durante o período do estudo, além da Região Sul, que apresenta a maior proporção de leitos públicos e a menor taxa de mortalidade durante esse período. Isso demonstra a importância das políticas públicas de saúde na distribuição igualitária e proporcional dos leitos, tendo em vista que a distribuição dos leitos/habitantes é bem heterogênea ao comparar os estados do país (NORONHA KVMS, et al., 2020).

O teste de associação de Spearman apresentou uma associação entre a distribuição dos leitos com a mortalidade. A Região Centro-Oeste, que apesar de apresentar o segundo maior número total de leitos, apresenta o segundo menor número de leitos SUS e, com isso, revelou-se a região com a maior taxa de mortalidade até o fim do estudo. Além disso, a Região Sul, que, ao contrário da Região Centro-Oeste, apresentou uma proporção maior de leitos públicos em relação aos privados, e, com isso, apresentou a menor taxa de mortalidade durante todo o estudo. Sendo assim, conforme assinalado por Pires LN, et al. (2020), as políticas de saúde pública se mostram fundamentais na distribuição equivalente e universal dos leitos pelas regiões do país, interferindo diretamente no prognóstico da saúde pública de cada região.

Foi observado uma relação entre a incidência e a letalidade, porém no início da pandemia todas as regiões apresentaram aumento na letalidade, enquanto que a curva da incidência estava com uma alta ainda insidiosa. Essa discrepância entre as curvas de incidência e letalidade pode ser devido ao alto índice de subnotificação, principalmente no início da pandemia no país (SANTOS W, et al., 2020).

Segundo Prado MF, et al. (2020), estudo realizado no início da pandemia, no Brasil as notificações eram de aproximadamente $8,0 \%$, ou seja, mais de $90 \%$ dos casos eram subnotificados. Estudos realizados na China e no Brasil mostraram que o índice de subnotificação era de $86 \%$ e próximo a $100 \%$, respectivamente. Essa falha no mapeamento e na notificação dos casos dificulta no delineamento preciso das características epidemiológicas da COVID-19 (NOGUEIRA AL, et al., 2020; DIAS GH, et al., 2020).

Apesar da condução cuidadosa deste estudo, vale a pena ressaltar que as limitações relacionadas ao próprio delineamento ecológico, que faz uso de informações de base de dados secundários, com coleta e alimentação de dados em constante atualização, determinada pela pandemia em curso, podem subestimar alguma informação epidemiológica relevante. Além disso, a subnotificação pode estar relacionada não só com a baixa testagem da população, mas também com a rápida disseminação e constante necessidade de atualização de forma atual e retrógrada dos números relativos a casos confirmados e óbitos.

Ademais, como o Censo de 2020 foi adiado devido à pandemia, os dados populacionais são referentes à estimativa populacional de 2019. Porém, esses são os dados mais recentes e utilizados como referência em todo o país, uma vez que a última versão do levantamento foi em 2010. Dessa forma, fica reconhecido as limitações relacionadas ao estudo, ainda que ele tenha sido produzido levando em consideração o delineamento e a interpretação cuidadosa dos dados levantados, com intuito de evitar potenciais vieses ou falácias, que podem ser uma das características dos estudos ecológicos.

\section{CONCLUSÃO}

Houve associação positiva entre o número de casos e a densidade demográfica, o que demonstra a relação direta existente entre a velocidade de disseminação da doença e a quantidade de pessoas habitando a mesma região. Além disso, também houve relação positiva entre a taxa de mortalidade e número de leitos por habitante. A distribuição desigual de leitos públicos e privados no Brasil pode sobrecarregar o SUS e prejudicar a qualidade do atendimento prestado. Esse estudo demonstra a importância de políticas de saúde pública eficazes para que ocorra uma distribuição igualitária dos investimentos destinados às regiões, bem como a aplicação correta deste capital financeiro por parte dos gestores dos estados. 


\section{REFERÊNCIAS}

1. BRASIL. Instituto Brasileiro de Geografia e Estatística. Análise da disponibilidade domiciliar de alimentos e do estado nutricional no Brasil. 2010. Disponível em: https://biblioteca.ibge.gov.br/visualizacao/livros/liv47310.pdf. Acesso em: 20 mar. 2020.

2. BRASIL. ANVISA. Nota Técnica no 04/2020: Orientações para serviços de saúde: medidas de prevenção e controle que devem ser adotadas durante a assistência aos casos suspeitos ou confirmados de infecção pelo novo

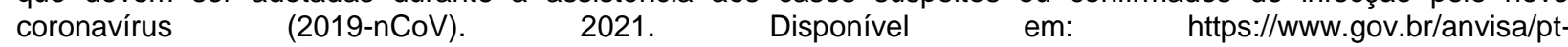
br/centraisdeconteudo/publicacoes/servicosdesaude/notas-tecnicas/nota-tecnica-gvims_ggtes_anvisa-04_2020-2502-para-o-site.pdf. Acesso em: 23 mar. 2021.

3. DIAS GH et. al. Análise da distribuição espacial da Covid-19 e subnotificação de casos novos e óbitos no estado do Rio Grande do Norte, Brasil. PGEO, 2020; 3(2): 51-67.

4. FARIAS HS. O avanço da Covid-19 e o isolamento social como estratégia para redução da vulnerabilidade. Revista Brasileira de Geografia Econômica: Espaço e Economia, 2020.

5. FRANCÊS CRL. Análise da evolução da pandemia de COVID-19 no Brasil - O Estado do Pará. Laboratório de Tecnologias Sociais. Revista Brasileira de Estudos de População. Belém-PA: UFPA, 2020.

6. FREITAS CM et. al. Covid-19 as a global disaster: Challenges to risk governance and social vulnerability in Brazil. Ambient. soc, 2020; 23: e0115.

7. GARCIA LP, DUARTE E. Intervenções não farmacológicas para o enfrentamento à epidemia da COVID-19 no Brasil. Epidemiol. Serv. Saúde, 2020; 29 (2): e2020222.

8. GOUVEIA GPM et. al. Incidência e letalidade da Covid-19 no Ceará, 2020. Revista Científica da Escola de Saúde Pública do Ceará: Cadernos ESP, 2020; 14.

9. HONGZHOU L et. al. Outbreak of pneumonia of unknown etiology in Wuhan, China: The mystery and the miracle. Journal of Medical Virology, 2020; Special Issue: 401-402.

10. HUI DS et. al. The continuing 2019-nCoV epidemic threat of novel coronaviruses to global health - The latest 2019 novel coronavirus outbreak in Wuhan, China. International Journal of Infectious Diseases, 2020; 91:264-266.

11. IBM Corp. 2017. IBM SPSS Statistics for Windows, Version 25.0. Armonk, NY: IBM Corp.

12. INLOCO. 2020. Mapa brasileiro da COVID-19. Brasil: Inloco. Disponível em: https://mapabrasileirodacovid.inloco.com.br/pt/.

13. JÚNIOR CAM. Covid-19 e isolamento social: algumas reflexões. Revista Augustus, 2020; 25:381-393.

14. KUCHARSKI AJ et al. Early dynamics of transmission and control of COVID-19: a mathematical modelling study. Lancet Infect Dis, 2020.

15. LANA CNA et. al. Determinantes Sociais da Saúde e Óbitos por Covid-19 nos Estados da Região Nordeste do Brasil. Rev. Bras. Saúde Funcional, 2020; 11(1): 18-29.

16. LEIVA GC et. al. Estrutura urbana e mobilidade populacional: implicações para o distanciamento social e disseminação da Covid-19. Revista Brasileira de Estudos de População, 2020; 37.

17. NOGUEIRA AL et. al. Estimativa da subnotificação de casos da Covid-19 no estado de Santa Catarina. Santa Catarina: UFSC, 2020.

18. NORONHA KVMS et. al. Pandemia por COVID-19 no Brasil: análise da demanda e da oferta de leitos hospitalares e equipamentos de ventilação assistida segundo diferentes cenários. Cad. Saúde Pública, 2020; 36(6): e00115320.

19. OLIVEIRA WK et. al. Como o Brasil pode deter a COVID-19. Epidemiol. Serv. Saúde, 2020; 29(2): e2020044.

20. OPAS/OMS. Folha informativa COVID-19 - Escritório da OPAS e da OMS no Brasil. 2020. Disponível em: https://www.paho.org/pt/covid19. Acesso em: 23 mar. 2021.

21. PEREIRA MD. O isolamento social em tempos de Covid-19 e seu paradoxo com os grupos em vulnerabilidade social. CGCHS, 2020; 6(2): 239.

22. PERES AC. Favelas contra o vírus: como as periferias vêm lidando com a pandemia de Covid-19, em meio aos problemas cotidianos e diante da ausência de ações governamentais: RADIS: Comunicação e Saúde, 2020; 212, 20-25.

23. PIRES LN et. al. COVID-19 e desigualdade: a distribuição dos fatores de risco no Brasil. Revista Brasileira de Geografia Econômica: Espaço e Economia, 2020.

24. PRADO MF et. al. Análise da subnotificação de COVID-19 no Brasil. Rev. bras. ter. intensiva, 2020; $32(2): 224-228$.

25. SANTOS $W$ et. al. Characterization of the COVID-19 pandemic and the impact of uncertainties, mitigation strategies, and underreporting of cases in South Korea, Italy, and Brazil. Chaos, Solitons \& Fractals, 2020; 136.

26. ZHANG CH, SCHWARTZ GG. Spatial Disparities in Coronavirus Incidence and Mortality in the United States: An Ecological Analysis as of May 2020. The Journal of Rural Health, 2020; 36:433-445. 